\title{
Nil 3-Armendariz Rings
}

\author{
Eltiyeb Ali ${ }^{1,2}$, Ayoub Elshokry ${ }^{1,2}$, Liu Zhongkui ${ }^{1}$ \\ ${ }^{1}$ Department of Mathematics, Northwest Normal University, Lanzhou, China \\ ${ }^{2}$ Department of Mathematics, Khartoum University, Omdurman, Sudan \\ Email: eltiyeb76@gmail.com, ayou1975@yahoo.com, liuzk@nwnu.edu.cn
}

Received November 13, 2013; revised December 13, 2013; accepted December 18, 2013

Copyright (C) 2013 Eltiyeb Ali et al. This is an open access article distributed under the Creative Commons Attribution License, which permits unrestricted use, distribution, and reproduction in any medium, provided the original work is properly cited. In accordance of the Creative Commons Attribution License all Copyrights (c) 2013 are reserved for SCIRP and the owner of the intellectual property Eltiyeb Ali et al. All Copyright (C) 2013 are guarded by law and by SCIRP as a guardian.

\begin{abstract}
We introduce nil 3-Armendariz rings, which are generalization of 3-Armendariz rings and nil Armendaiz rings and investigate their properties. We show that a ring $R$ is nil 3-Armendariz ring if and only if for any $n \in \mathbb{N}, T_{n}(R)$ is nil 3-Armendariz ring. Also we prove that a right Ore ring $R$ is nil 3-Armendariz if and only if so is $Q$, where $Q$ is the classical right quotient ring of $R$. With the help of this result, we can show that a commutative ring $R$ is nil 3-Armendariz if and only if the total quotient ring of $R$ is nil 3-Armendariz.
\end{abstract}

Keywords: Armendariz Ring; 3-Armendariz Ring; Nil Armendariz Ring; Nil 3-Armendariz Ring

\section{Introduction}

Throughout this article, $R$ denotes an associative ring, not necessary with identity. Given a ring $R$, the polynomial ring over $R$ is denoted by $R[x]$. The study of Armendariz ring was initiated by Armendariz [1] and Rege and Chhawchharia [2]. A ring $R$ is called Armendariz if whenever polynomials

$f(x)=a_{0}+a_{1} x+\cdots+a_{n} x^{n}$,

$g(x)=b_{0}+b_{1} x+\cdots+b_{m} x^{m} \in R[x]$ satisfy

$f(x) g(x)=0$, then $a_{i} b_{j}=0$, for all $i, j$. (The converse is always true.) Some properties of Armendariz rings have been studied in Rege and Chhawchharia [2], Anderson and Camillo [3], Kim and Lee [4], Huh et al. [5], and Lee and Wong [6]. Suiyi [7] introduced the notion of 3-Armendariz ring. A ring $R$ is called a 3-Armendariz if whenever polynomials

$$
\begin{aligned}
& f(x)=a_{0}+a_{1} x+\cdots+a_{n} x^{n}, \\
& g(x)=b_{0}+b_{1} x+\cdots+b_{m} x^{m}, \\
& h(x)=c_{0}+c_{1} x+\cdots+c_{r} x^{r} \in R[x] \text { satisfy } \\
& f(x) g(x) h(x)=0, \text { then } a_{i} b_{j} c_{k}=0, \text { for all } i, j, k .
\end{aligned}
$$$$
\text { Due to Ramon Antoine [8], a ring } R \text { is said to be nil }
$$$$
\text { Armendariz if whenever two polynomials }
$$$$
f(x), g(x) \in R[x] \text {, satisfy } f(x) g(x) \in \operatorname{nil}(R)[x]
$$$$
\text { then } a b \in \operatorname{nil}(R) \text {, for all } a \in \operatorname{coef}(f(x)) \text { and }
$$

$b \in \operatorname{coef}(g(x))$. There is a nil Armendariz ring but not Armendaiz by [8, Example 4.11]. A ring $R$ is called reduced if it has no nonzero nilpotent elements. Armendariz rings are thus a generalization of reduced rings, and therefore, nilpotent elements play an important role in this class of rings. There are many examples of rings with nilpotent elements which are Armendariz. In fact, in [3], Anderson and Camillo prove that if $n \geq 2$, then $R[x] /\left(x^{n}\right)$ is an Armendariz ring if and only if $R$ is reduced. In [9], Liu and Zhao introduced weak Armendariz rings as a generalization of Armendariz rings. A ring is weak Armendariz if whenever the product of two polynomials is zero then the product of their coefficients is nilpotent. In [10], Wu Hui-feng introduced the concept of weak 3-Armendariz ring as a generalization of 3-Armendariz rings and weak Armendariz ring and investigated their properties. A ring is weak 3-Armendariz if whenever the product of three polynomials is zero then the product of their coefficients is nilpotent. Motivated by results in Suiyi [7], Liu and Zhao [9], Antoine [8], Kim and Lee [4], Rege and Chhawchharia [2], and $\mathrm{Wu}$ Hui-feng $[10,11]$, we investigate a generalization of nil Armendariz rings and 3-Armemdariz rings which we call nil 3-Armendariz rings.

\section{Nil 3-Armendariz Rings}

If $R$ is a ring, $\operatorname{nil}(R)$ denotes the set of all nilpotent elements in $R$, and if $f(x) \in R[x], \quad \operatorname{coe} f(f(x))$ 
denotes the subset of $R$ of the coefficients of $f(x)$.

Condition (P): For all $a, b, c \in R$, if $(a b c)^{2}=0$, then $a b c=0 . \quad$ ( See [7])

Lemma 2.1. [11, Proposition 1]. If $R$ is a reduced ring, then $R$ satisfies the condition (P), but the converse is not true.

Lemma 2.2. [7, Theorem 1]. If a ring $R$ satisfies condition (P), then $R$ is a 3-Armendariz ring.

Proposition 2.3. Let $R$ be a ring such that $\operatorname{nil}(R) \unlhd R$. If $f(x) g(x) h(x) \in \operatorname{nil}(R)[x]$, then $a b c \in \operatorname{nil}(R)$ for all $a \in$ coef $(f(x))$, $b \in \operatorname{coef}(g(x))$ and $c \in$ coef $(h(x))$.

Proof. Observe that $R / \operatorname{nil}(R)$ is reduced. By Lemma 2.1, $R / \operatorname{nil}(R)$ satisfies condition (P) and by Lemma 2.2, $R / \operatorname{nil}(R)$ is 3-Armendariz. Suppose $\underline{f}(x) g(x) h(x) \in \operatorname{nil}(R)[x]$. Then, if we denote by $\bar{f}(x), \bar{g}(x), \bar{h}(x)$ the corresponding polynomials in $R / \operatorname{nil}(R)[x], \quad f(x) \bar{g}(x) \bar{h}(x)=\overline{0}$. Since $R / \operatorname{nil}(R)$ is 3-Armendariz, $\bar{a} b \bar{c}=0$ for all $\bar{a} \in \operatorname{coef}(\bar{f}(x))$, $\bar{b} \in \operatorname{coef}(\bar{g}(x))$, and $\bar{c} \in \operatorname{coef}(\bar{h}(x))$. Hence $a b c$ is nil for all $a \in \operatorname{coef}(f(x)), \quad b \in \operatorname{coef}(g(x))$ and $c \in$ coef $(h(x))$.

$\mathrm{Wu}$ Hui-feng gives the following generalization of 3-Armendariz rings.

Definition 2.4. [10, Definition 1]. A ring $R$ is said to be a weak 3-Armendariz ring if whenever polynomials $f(x), g(x), h(x) \in R[x]$, satisfy $f(x) g(x) h(x)=0$, then $a b c \in \operatorname{nil}(R)$ for all

$a \in$ coef $(f(x)), b \in$ coef $(g(x))$ and $c \in$ coef $(h(x))$.

Clearly, 3-Armendariz rings are weak 3-Armendariz.

We now present here a stronger condition, given by the property obtained in Proposition 2.3.

Definition 2.5. A ring $R$ is said to be nil 3-Armendariz if whenever polynomials

$f(x), g(x), h(x) \in R[x]$, satisfy

$f(x) g(x) h(x) \in \operatorname{nil}(R)[x]$ then $a b c \in \operatorname{nil}(R)$ for all $a \in$ coef $(f(x)), \quad b \in \operatorname{coef}(g(x))$ and

$c \in$ coef $(h(x))$.

Observe that if $\operatorname{nil}(R) \unlhd R$ then by Proposition 2.3,

$R$ is nil 3-Armendariz. More generally we obtain the following.

Proposition 2.6. Let $R$ be a ring that satisfies the condition (P), and $I \triangleleft R$ a nil ideal. Then $R$ is nil 3-Armendariz if and only if $R / I$ is nil 3-Armendariz.

Proof. We denote $\bar{R}=R / I$. Since $I$ is nil, then $\operatorname{nil}(\bar{R})=\operatorname{nil}(R)$. Hence $f(x) g(x) h(x) \in \operatorname{nil}(R)[x]$ if and only if $\bar{f}(x) \bar{g}(x) \bar{h}(x) \in \operatorname{nil}(\bar{R})[x]$. And, if $a \in$ coef $(f(x)), \quad b \in \operatorname{coef}(g(x))$ and $c \in \operatorname{coef}(h(x))$ then $a b c \in \operatorname{nil}(R)$ if and only if $\bar{a} \bar{b} \bar{c} \in \operatorname{nil}(\bar{R})$. Therefore $R$ is nil 3-Armendariz if and only if $\bar{R}$ is nil 3-Armendariz.

The next results can be proved by using the technique used in the proof of [8, Lemma 2.5, Lemma 2.6].

Lemma 2.7. Let $R$ be a nil 3-Armendariz ring and $n \geq 3$. If $f_{1}(x), f_{2}(x), \cdots, f_{n}(x) \in R[x]$ such that $f_{1}(x) f_{2}(x) \cdots f_{n}(x) \in \operatorname{nil}(R)[x]$, then if $a_{k} \in$ coef $\left(f_{k}(x)\right)$ for $k=1, \cdots, n$, we have $a_{1} a_{2} \cdots a_{n} \in \operatorname{nil}(R)$.

Lemma 2.8. If $R$ is a 3-Armendariz ring then $\operatorname{nil}(R)[x] \subseteq \operatorname{nil}(R[x])$.

Proposition 2.9. If $R$ is a 3-Armendariz ring then $R$ is nil 3-Armendariz.

Proof. Suppose $f(x), g(x), h(x) \in R[x]$ be such that $f(x) g(x) h(x) \in \operatorname{nil}(R)[x]$. Since $R$ is $3-\operatorname{Ar}-$ mendariz, by Lemma 2.8, $f(x) g(x) h(x)$ is nilpotent and there exists $n \geq 1$ such that $(f(x) g(x) h(x))^{n}=0$. Hence, since $R$ is 3-Armendariz, for all $a \in \operatorname{coef}(f(x))$,

$b \in \operatorname{coef}(g(x))$ and $c \in \operatorname{coef}(h(x))$ by choosing the corresponding coefficient in each polynomial, we have $a b c a b c \cdots a b c=0$ and thus, $a b c \in \operatorname{nil}(R)$. Therefore $R$ is nil 3-Armendariz.

Proposition 2.10. The class of nil 3-Armendariz rings is closed under finite direct products.

Proof. Let $R=\prod_{s \in \beta} R_{s}$ be the finite direct product of $R_{s}$ where $\beta=\{1,2, \cdots, p\}, \quad R_{s}$ is nil 3-Armendariz. Suppose $f(x) g(x) h(x)=0$ for some polynomials $f(x)=a_{0}+a_{1} x+\cdots+a_{n} x^{n}$,

$g(x)=b_{0}+b_{1} x+\cdots+b_{m} x^{m}$,

$h(x)=c_{0}+c_{1} x+\cdots+c_{r} x^{r} \in R[x]$, where $a_{i}=\left(a_{i 1}, a_{i 2}, \cdots, a_{i p}\right), \quad b_{j}=\left(b_{j 1}, b_{j 2}, \cdots, b_{j p}\right)$,

$c_{k}=\left(c_{k 1}, c_{k 2}, \cdots, c_{k p}\right)$, are elements of the product ring

$R$. Set $f_{s}(x)=\sum_{i=0}^{n} a_{i s} x^{i}, \quad g_{s}(x)=\sum_{j=0}^{m} b_{j s} x^{j} \quad$ and $h_{s}(x)=\sum_{k=0}^{r} c_{k s} x^{k} \in R[x]$. Since $f(x) g(x) h(x)=0$ then $\sum_{i+j+k=l} a_{i} b_{j} c_{k}=0, \quad 0 \leq l \leq n+m+r$. So

$\sum_{i+j+k=l}\left(a_{i 1} b_{j 1} c_{k 1}, \cdots, a_{i p} b_{j p} c_{k p}\right)=0$, and so

$\sum_{i+j+k=l}\left(a_{i s} b_{j s} c_{k s}\right)=0,1 \leq s \leq p$. Thus

$f_{s}(x) g_{s}(x) h_{s}(x)=0$ in $R_{s}[x], 1 \leq s \leq p$. Since $R_{s}$ is nil 3-Armendariz, then we have $a_{i s} b_{j s} c_{k s} \in \operatorname{nil}\left(R_{s}\right)$. Now, for each $i, j, k$, there exist positive integers $m_{i j k s}$ such that $\left(a_{i s} b_{j s} c_{k s}\right)^{m_{i j k s}}=0$, in the ring $R_{s}, \quad 1 \leq s \leq p$. If we take $m_{i j k}=\max \left\{m_{i j k s}: 1 \leq s \leq p\right\}$, then it is clear that $\left(a_{i s} b_{j s} c_{k s}\right)^{m_{i j k}}=0$. Therefore $a_{i} b_{j} c_{k} \in \operatorname{nil}(R)$. This means that $R$ is nil 3-Armendariz.

Lemma 2.11. Let $S$ be a subring of $R$. If $R$ is nil 3-Armendariz. Then so is $S$.

Proof. Let $f(x), g(x), h(x) \in S[x]$, be such that $f(x) g(x) h(x) \in \operatorname{nil}(S[x])$. Then $f(x) g(x) h(x) \in \operatorname{nil}(R[x])$. Since $R$ is nil 3-Armendariz, then $a_{i} b_{j} c_{k} \in \operatorname{nil}(R)$, i.e., $\left(a_{i} b_{j} c_{k}\right)^{n_{j k}}=0 \Rightarrow a_{i} b_{j} c_{k} \in \operatorname{nil}(S)$. This means that $S$ is nil 3-Armendariz. 
We denote by $T_{n}(R)$ the ring consisting of all n-by-n upper triangular matrices over $R$. In [10, Theorem 1], showed that $R$ is a weak 3-Armendariz if and only if $T_{n}(R)$ is a weak 3 -Armendariz ring for all $n \in \mathbb{N}$. Here we have a similar results for nil 3-Armendariz rings.

Proposition 2.12. Let $R$ be a ring. The following conditions are equivalent:

1) $R$ is nil 3-Armendariz;

2) for any $n, T_{n}(R)$ is nil 3-Armendariz.
Proof. (2) $\Rightarrow(1)$ We note that any subring of nil 3-Armendariz rings is nil 3- Armendariz by Lemma 2.11. Thus if $T_{n}(R)$ is nil 3-Armendariz ring, then so is $R$. (1) $\Rightarrow(2)$ Let $f(x)=A_{0}+A_{1} x+\cdots+A_{p} x^{p}$, $g(x)=B_{0}+B_{1} x+\cdots+B_{q} x^{q}$, and $h(x)=C_{0}+C_{1} x+\cdots+C_{d} x^{d}$ be elements of $T_{n}(R)[x]$. It is easy to see that there exists an isomorphism of rings $T_{n}(R)[x] \rightarrow T_{n}(R[x])$ define by:

$$
\sum_{i=0}^{p}\left(\begin{array}{ccccc}
a_{11}^{i} & a_{12}^{i} & a_{13}^{i} & \cdots & a_{1 n}^{i} \\
0 & a_{22}^{i} & a_{23}^{i} & \cdots & a_{2 n}^{i} \\
0 & 0 & a_{33}^{i} & \cdots & a_{3 n}^{i} \\
\vdots & \vdots & \vdots & \ddots & \vdots \\
0 & 0 & 0 & \cdots & a_{n n}^{i}
\end{array}\right) x^{i} \mapsto\left(\begin{array}{ccccc}
\sum_{i=0}^{p} a_{11}^{i} x^{i} & \sum_{i=0}^{p} a_{12}^{i} x^{i} & \sum_{i=0}^{p} a_{13}^{i} x^{i} & \cdots & \sum_{i=0}^{p} a_{1 n}^{i} x^{i} \\
0 & \sum_{i=0}^{p} a_{22}^{i} x^{i} & \sum_{i=0}^{p} a_{23}^{i} x^{i} & \cdots & \sum_{i=0}^{p} a_{2 n}^{i} x^{i} \\
0 & 0 & \sum_{i=0}^{p} a_{33}^{i} x^{i} & \cdots & \sum_{i=0}^{p} a_{3 n}^{i} x^{i} \\
\vdots & \vdots & \vdots & \ddots & \vdots \\
0 & 0 & 0 & \cdots & \sum_{i=0}^{p} a_{n n}^{i} x^{i}
\end{array}\right) .
$$

Assume that $f(x) g(x) h(x) \in \operatorname{nil}\left(T_{n}(R)[x]\right)$. Let

$$
A_{i}=\left(\begin{array}{ccccc}
a_{11}^{i} & a_{12}^{i} & a_{13}^{i} & \cdots & a_{1 n}^{i} \\
0 & a_{22}^{i} & a_{23}^{i} & \cdots & a_{2 n}^{i} \\
0 & 0 & a_{33}^{i} & \cdots & a_{3 n}^{i} \\
\vdots & \vdots & \vdots & \ddots & \vdots \\
0 & 0 & 0 & \cdots & a_{n n}^{i}
\end{array}\right), B_{j}=\left(\begin{array}{ccccc}
b_{11}^{j} & b_{12}^{j} & b_{13}^{j} & \cdots & b_{1 n}^{j} \\
0 & b_{22}^{j} & b_{23}^{j} & \cdots & b_{2 n}^{j} \\
0 & 0 & a_{33}^{j} & \cdots & b_{3 n}^{j} \\
\vdots & \vdots & \vdots & \ddots & \vdots \\
0 & 0 & 0 & \cdots & b_{n n}^{j}
\end{array}\right) \text { and } C_{k}=\left(\begin{array}{ccccc}
c_{11}^{k} & c_{12}^{k} & c_{13}^{k} & \cdots & c_{1 n}^{k} \\
0 & c_{22}^{k} & c_{23}^{k} & \cdots & c_{2 n}^{k} \\
0 & 0 & c_{33}^{k} & \cdots & c_{3 n}^{k} \\
\vdots & \vdots & \vdots & \ddots & \vdots \\
0 & 0 & 0 & \cdots & c_{n n}^{k}
\end{array}\right) \text {. }
$$

Then

$$
\begin{aligned}
& \left(\begin{array}{ccccc}
\sum_{i=0}^{p} a_{11}^{i} x^{i} & \sum_{i=0}^{p} a_{12}^{i} x^{i} & \sum_{i=0}^{p} a_{13}^{i} x^{i} & \cdots & \sum_{i=0}^{p} a_{1 n}^{i} x^{i} \\
0 & \sum_{i=0}^{p} a_{22}^{i} x^{i} & \sum_{i=0}^{p} a_{23}^{i} x^{i} & \cdots & \sum_{i=0}^{p} a_{2 n}^{i} x^{i} \\
0 & 0 & \sum_{i=0}^{p} a_{33}^{i} x^{i} & \cdots & \sum_{i=0}^{p} a_{3 n}^{i} x^{i} \\
\vdots & \vdots & \vdots & \ddots & \vdots \\
0 & 0 & 0 & \cdots & \sum_{i=0}^{p} a_{n n}^{i} x^{i}
\end{array}\right) \\
& \times\left(\begin{array}{cccccc}
\sum_{j=0}^{q} b_{11}^{j} x^{j} & \sum_{j=0}^{q} b_{12}^{j} x^{j} & \sum_{j=0}^{q} b_{13}^{j} x^{j} & \cdots & \sum_{j=0}^{q} b_{1 n}^{j} x^{j} \\
0 & \sum_{j=0}^{q} b_{22}^{j} x^{j} & \sum_{j=0}^{q} b_{23}^{j} x^{j} & \cdots & \sum_{j=0}^{q} b_{2 n}^{j} x^{j} \\
0 & 0 & \sum_{j=0}^{q} b_{33}^{j} x^{j} & \cdots & \sum_{j=0}^{q} b_{3 n}^{j} x^{j} \\
\vdots & \vdots & \vdots & \ddots & \vdots \\
0 & 0 & 0 & \cdots & \sum_{j=0}^{q} b_{n n}^{j} x^{j}
\end{array}\right) \\
& \times\left(\begin{array}{ccccc}
\sum_{k=0}^{d} c_{11}^{k} x^{k} & \sum_{k=0}^{d} c_{12}^{k} x^{k} & \sum_{k=0}^{d} c_{13}^{k} x^{k} & \cdots & \sum_{k=0}^{d} c_{1 n}^{k} x^{k} \\
0 & \sum_{k=0}^{d} c_{22}^{k} x^{k} & \sum_{k=0}^{d} c_{23}^{k} x^{k} & \cdots & \sum_{k=0}^{d} c_{2 n}^{k} x^{k} \\
0 & 0 & \sum_{k=0}^{d} c_{33}^{k} x^{k} & \cdots & \sum_{k=0}^{d} c_{3 n}^{k} x^{k} \\
\vdots & \vdots & \vdots & \ddots & \vdots \\
0 & 0 & 0 & \cdots & \sum_{k=0}^{d} c_{n n}^{k} x^{k}
\end{array}\right)
\end{aligned}
$$

corresponds a polynomial with coefficients in nil $\left(T_{n}(R)\right)$ under the isomorphism 
$T_{n}(R)[x] \rightarrow T_{n}(R[x])$. Because $T_{n}(R)[x] \cong T_{n}(R[x])$ and

$$
\operatorname{nil}\left(T_{n}(R)\right)=\left(\begin{array}{cccc}
n l(R) i & R & R & R \\
0 & \operatorname{nil}(R) & R & R \\
\vdots & \vdots & \ddots & \vdots \\
0 & 0 & \cdots & \operatorname{nil}(R)
\end{array}\right),
$$

$$
\left(A_{i} B_{j} C_{k}\right)^{m_{i j k}}=\left(\begin{array}{c}
\left(a_{11}^{i} b_{11}^{j} c_{11}^{k}\right)^{m_{i j k}} \\
0 \\
\vdots \\
0
\end{array}\right.
$$$$
\left(a_{22}^{i} b_{22}^{j} c_{22}^{k}\right)^{m_{i j k}}
$$

Thus, $\left(\left(A_{i} B_{j} C_{k}\right)^{m_{i j k}}\right)^{n}=0$ and so

$A_{i} B_{j} C_{k} \in \operatorname{nil}\left(T_{n}(R)\right)$. This shows that $T_{n}(R)$ is nil 3-Armendariz.

Corollary 2.13. If $R$ is a 3-Armendariz ring, then, for any $n, T_{n}(R)$ is nil 3-Armendariz ring.

In [10, Corollary 1], it is shown that a ring is a weak 3-Armendariz ring if and only if $R[x] /\left(x^{n}\right)$ is a weak 3-Armendariz ring, where $\left(x^{n}\right)$ is the ideal of $R[x]$ generated by $x^{n}$ and $n$ is a positive integer. For nil 3-Armendariz rings, we have the following result.

Proposition 2.14. Let $R$ be a ring and $n$ any positive integer. Then $R$ is nil 3-Armendariz if and only if $R[x] /\left(x^{n}\right)$ is nil 3-Armendariz, where $\left(x^{n}\right)$ is the ideal of $R[x]$ generated by $x^{n}$.

Proof. As $R[x] /\left(x^{n}\right) \cong S$ where

$$
S=\left\{\left(\begin{array}{cccc}
a_{0} & a_{1} & \cdots & a_{n-1} \\
0 & a_{0} & \cdots & a_{n-2} \\
\cdots & \cdots & \ddots & \cdots \\
0 & 0 & \cdots & a_{0}
\end{array}\right) \mid a_{i} \in R, i=0,1, \cdots, n-1\right\}
$$

is a subring of $T_{n}(R)$. If $R$ is nil 3-Armendariz, then, by Proposition 2.12, we have that $T_{n}(R)$ is nil 3-Armendariz, and so is $S$. Thus, $R[x] /\left(x^{n}\right)$ is nil 3-Armendariz. Conversely, if $R[x] /\left(x^{n}\right)$ is nil 3-Armen- dariz, then $R$ as a subring of $R[x] /\left(x^{n}\right)$, is nil 3- Armendariz too.

Corollary 2.15. A ring $R$ is nil 3-Armendariz if and only if the trivial extension $T(R, R)$ is nil 3-Armendariz.

Proof. It follows from Proposition 2.12.

From Proposition 2.12, one may suspect that if $R$ is nil 3-Armendariz then every n-by-n full matrix ring $M_{n}(R)$ over $R$ is nil 3-Armendariz, where $n \geq 2$. But the following example erases the possibility.

Example 2.16. Let $R$ be a ring and let we have

$$
\left(\sum_{i=0}^{p} a_{s s}^{i} x^{i}\right)\left(\sum_{j=0}^{q} b_{s s}^{j} x^{j}\right)\left(\sum_{k=0}^{d} c_{s s}^{k} x^{k}\right) \in n \operatorname{ll}(R)[x],
$$

for $s=1,2, \cdots, n$.

Since $R$ is nil 3-Armendariz, there exists $m_{i j k s} \in \mathbb{N}$ such that $\left(a_{s s}^{i} b_{s s}^{j} c_{s s}^{k}\right)^{m_{i j k s}}=0$ for any $s$ and any $i, j, k$. Let $m_{i j k}=\max \left\{m_{i j k 1}, m_{i j k 2}, \cdots, m_{i j k n}\right\}$. Then

$$
\left.\begin{array}{cc}
\ldots & * \\
\ldots & * \\
\ddots & * \\
\ldots & \left(a_{n n}^{i} b_{n n}^{j} c_{n n}^{k}\right)^{m_{i j k}}
\end{array}\right)=\left(\begin{array}{cccc}
0 & * & \ldots & * \\
0 & 0 & \ldots & * \\
\vdots & \vdots & \ddots & * \\
0 & 0 & \cdots & 0
\end{array}\right) .
$$

$S=M_{2}(F)$. Let

$$
\begin{aligned}
& f(x)=\left(\begin{array}{ll}
0 & 1 \\
0 & 0
\end{array}\right)+\left(\begin{array}{ll}
1 & 0 \\
0 & 0
\end{array}\right) x, \\
& g(x)=\left(\begin{array}{ll}
1 & 0 \\
0 & 1
\end{array}\right) x, h(x)=\left(\begin{array}{ll}
1 & 1 \\
0 & 0
\end{array}\right)+\left(\begin{array}{cc}
0 & 0 \\
-1 & -1
\end{array}\right) x
\end{aligned}
$$

be polynomials in $S[x]$. Then $f(x) g(x) h(x)=0$. But

$$
\left(\begin{array}{ll}
1 & 0 \\
0 & 0
\end{array}\right)\left(\begin{array}{ll}
1 & 0 \\
0 & 1
\end{array}\right)\left(\begin{array}{ll}
1 & 1 \\
0 & 0
\end{array}\right)=\left(\begin{array}{ll}
1 & 1 \\
0 & 0
\end{array}\right)
$$

is not nilpotent. Thus $S$ is not nil 3-Armendariz. Now we can give the example of nil 3-Armendariz rings which are not 3-Armendariz.

Example 2.17. Let $R$ be a nil 3-Armendariz ring. Then the ring

$$
R_{n}=\left\{\left(\begin{array}{ccccc}
a & a_{12} & a_{13} & \cdots & a_{1 n} \\
0 & a & a_{23} & \cdots & a_{2 n} \\
0 & 0 & a & \cdots & a_{3 n} \\
\vdots & \vdots & \vdots & \ddots & \vdots \\
0 & 0 & 0 & \cdots & a
\end{array}\right) \mid a, a_{i j} \in R\right\}
$$

is not 3-Armendariz by [7, Example 4], for $n \geq 4$, but $R_{n}$ is a nil 3-Armendariz ring by Proposition 2.12, because $R_{n}$ is a subring of $T_{n}(R)$.

Proposition 2.18. Let $R$ be a ring and $e$ an idempotent of $R$. If $e$ is central in $R$, then the following statements are equivalent:

1) $R$ is nil 3-Armendariz;

2) $e R$ and $(1-e) R$ are nil 3-Armendariz.

Proof. (2) $\Rightarrow(1)$. Is obvious since $e R$ and $(1-e) R$ are subrings of $R$.

(1) $\Rightarrow(2)$. Note that $R=e R \oplus(1-e) R$ as rings. Thus the result follows from Proposition 2.10.

In [5, Theorem 11], it was shown that if $I$ is a re- 
duced ideal of $R$ such that $R / I$ is Armendariz, then $R$ is Armendariz. In [10, Proposition 4], it is shown that if $R / I$ is a weak 3-Armendariz ring, then so is $R$, where $I$ is a nilpotent ideal of $R$. We show that this result also holds for nil 3-Armendariz rings in the following.

Proposition 2.19. Let $R$ be a ring such that $R / I$ is a nil 3-Armendariz ring for some proper ideal $I$ of $R$. If $I \subseteq \operatorname{nil}(R)$, then $R$ is nil 3-Armendariz.

Proof. Let $f(x)=\sum_{i=0}^{n} a_{i} x^{i}, \quad g(x)=\sum_{j=0}^{m} b_{j} x^{j}$,

$h(x)=\sum_{k=0}^{r} c_{k} x^{k} \in R[x]$ such that

$f(x) g(x) h(x) \in \operatorname{nil}(R)[x]$. Then

$\left(\sum_{i=0}^{n} \bar{a}_{i} x^{i}\right)\left(\sum_{j=0}^{m} \bar{b}_{j} x^{j}\right)\left(\sum_{k=0}^{r} \bar{c}_{k} x^{k}\right) \in \operatorname{nil}(R / I)[x]$.

Since $R / I$ is nil 3-Armendariz, we have that $\bar{a}_{i} \bar{b}_{j} \bar{c}_{k} \in \operatorname{nil}(R / I)$. Hence $a_{i} b_{j} c_{k} \in I$. Since $I \subseteq \operatorname{nil}(R)$, then $a_{i} b_{j} c_{k} \in \operatorname{nil}(R)$. This means that $R$ is a nil 3-Armendariz ring.

Anderson and Camillo in [3, Theorem 2], prove that a ring $R$ is Armendariz if and only if the polynomial ring $R[x]$ is Armendariz. Yang Suiyi [7], prove that a ring $R$ is 3-Armendariz if and only if the polynomial ring $R[x]$ is 3-Armendariz. In [11], it is shown that if $R$ is reduced ring, then $R[x]$ and $R[[x]]$ is 3-Armendariz ring. For nil 3-Armendariz rings we will give the following results.

Proposition 2.20. If $R$ is nil 3-Armendariz, then $\operatorname{nil}(R[x]) \subseteq \operatorname{nil}(R)[x]$.

Proof. Suppose $f(x) \in \operatorname{nil}(R[x])$ and $f(x)^{k}=0$. By Lemma 2.7, we have that $a_{0} \cdots a_{k} \in \operatorname{nil}(R)$ where $a_{i} \in \operatorname{coef}(f(x))$ for $i=1, \cdots, k$. In particular, for every $a \in$ coef $(f), a^{k}$ is nilpotent. Therefore $a \in \operatorname{nil}(R)$ for all $a \in \operatorname{coef}(f(x))$ and hence $f(x) \in \operatorname{nil}(R)[x]$.

Theorem 2.21. If $R$ is a 3-Armendariz ring, then $R[x]$ is a nil 3-Armendariz ring.

Proof. Let $R$ be 3-Armendariz ring. Then by [7, Theorem 3], $R[x]$ is 3-Armendariz. Thus by Proposition 2.9, $R[x]$ is nil 3-Armendariz.

Proposition 2.22. Let $R$ be a reduced ring. Then

$R[[x]]$ is a nil 3-Armendariz ring.

Proof. It follows from the method in the proof of [11, Theorem 1].

Corollary 2.23. If $R$ is a reduced ring, then $R[x]$ is a nil 3-Armendariz ring.

Recall that an element $u$ of a ring $R$ is right regular if $u r=0$ implies $r=0$ for $r \in R$. Similarly, left regular elements can be defined. An element is regular if it is both left and right regular (and hence not a zero divisor).

A ring $R$ is called right (resp., left) Ore if given $a, b \in R$ with $b$ regular, there exist $a_{1}, b_{1} \in R$ with $b_{1}$ regular such that $a b_{1}=b a_{1}\left(\right.$ resp., $\left.b_{1} a=a_{1} b\right)$. It is a well-known fact that $R$ is a right (resp., left) Ore ring if and only if the classical right (resp., left) quotient ring of $R$ exists.

Lemma 2.24. If $f(x) \in \operatorname{nil}(R)[x]$, then for any central element $\lambda \in R, \quad \lambda f(x) \in \operatorname{nil}(R)[x]$.

Proof. Set $f(x)=a_{0}+a_{1} x+\cdots+a_{n} x^{n}$. Then $a_{i} \in \operatorname{nil}(R)$. Thus $\lambda a_{i} \in \operatorname{nil}(R)$. This means that $\lambda f(x) \in \operatorname{nil}(R)[x]$.

Theorem 2.25. Let $R$ be a right Ore ring with the classical right quotient ring $Q$. If all right regular elements are central, then $R$ is nil 3-Armendariz if and only if so is $Q$.

Proof. It suffices to show by Lemma 2.11, that if $R$ is nil 3-Armendariz rings so is $Q$. We apply the proof of [5, Theorem 12]. Consider $f(x)=\sum_{i=0}^{n} \alpha_{i} x^{i}$, $g(x)=\sum_{j=0}^{m} \beta_{j} x^{j}, h(x)=\sum_{k=0}^{r} \gamma_{k} x^{k} \in Q[x]$, such that $f(x) g(x) h(x) \in \operatorname{nil}(Q)[x]$. By [12, Proposition 2.1.16], we can assume that $\alpha_{i}=a_{i} u^{-1}$, $\beta_{j}=b_{j} v^{-1}, \gamma_{k}=c_{k} w^{-1}$ with $a_{i}, b_{j}, c_{k} \in R$ for all $i, j, k$ and a right regular elements $u, v, w \in R$. Put $\phi(x)=\sum_{i=0}^{n} a_{i} x^{i}, \varphi(x)=\sum_{j=0}^{m} b_{j} x^{j}$, $\psi(x)=\sum_{k=0}^{r} c_{k} x^{k} \in R[x]$. Then we have

$$
\begin{aligned}
& f(x) g(x) h(x) \in \operatorname{nil}(Q)[x] \\
& =\sum_{p=0}^{n+m+r}\left(\sum_{i+j+k=p} \alpha_{i} \beta_{j} \gamma_{k}\right) x^{p} \\
& =\sum_{p=0}^{n+m+r}\left(\sum_{i+j+k=p} a_{i} u^{-1} b_{j} v^{-1} c_{k} w^{-1}\right) x^{p} \\
& =\sum_{p=0}^{n+m+r}\left(\sum_{i+j+k=p} a_{i} b_{j} c_{k}(u v w)^{-1}\right) x^{p} \\
& =\phi(x) \varphi(x) \psi(x)(u v w)^{-1} .
\end{aligned}
$$

Since $\phi(x) \varphi(x) \psi(x)(u v w)^{-1} \in \operatorname{nil}(R)[x]$, by Lemma 2.24, $\phi(x) \varphi(x) \psi(x) \in \operatorname{nil}(R)[x]$. Since $R$ is nil 3-Armendariz, $a_{i} b_{j} c_{k} \in \operatorname{nil}(R)$ for each $i, j, k$ and so

$\alpha_{i} \beta_{j} \gamma_{k}=a_{i} u^{-1} b_{j} v^{-1} c_{k} w^{-1}=a_{i} b_{j} c_{k}(u v w)^{-1} \in \operatorname{nil}(Q)$, for all $i, j, k$. Therefore $Q$ is nil 3-Armendariz ring.

Corollary 2.26. Let $R$ be a ring and $\Delta$ be a multiplicative closed subset in $R$ consisting of central regular elements. Then $R$ is nil 3-Armendariz rings if and only if $\Delta^{-1} R$ is nil 3-Armendariz rings.

Corollary 2.27. A commutative ring $R$ is nil 3-Armendariz if and only if so is the total quotient ring of $R$.

Proof. It suffices to show the necessity by Lemma 2.11. Let $\Delta$ be the multiplicative closed subset of all regular elements in $R$. Then $\Delta^{-1} R$ is the total quotient ring of $R$ and hence the result holds by Corollary 2.26.

The ring of Laurent polynomials in $X$, with coefficients in a ring $R$, consists of all formal sum 
$\sum_{i=k}^{n} m_{i} x^{i}$ with obvious addition and multiplication, where $m_{i} \in R$ and $k, n$ are (possibly negative) integers and denote it by $R\left[x ; x^{-1}\right]$.

Corollary 2.28. Let $R$ be a ring. $R[x]$ is nil 3Armendariz if and only if $R\left[x ; x^{-1}\right]$ is nil 3-Armendariz.

Proof. It suffices to establish necessity since $R[x]$ is a subring of $R\left[x ; x^{-1}\right]$. Let $\Delta=\left\{1, x, x^{2}, \cdots\right\}$, then clearly $\Delta$ is a multiplicatively closed subset in $R[x]$ consisting of central regular elements. Note that $R\left[x ; x^{-1}\right]=\Delta^{-1} R[x]$. If $R[x]$ is nil 3-Armendariz, so is $\Delta^{-1} R[x]$ by Corollary 2.26.

\section{Acknowledgements}

This paper is partially supported by National Natural Science Foundation of China (No.11261050). I also thank the referee for his or her valuable comments.

\section{REFERENCES}

[1] E. P. Armendariz, "A Note on Extensions of Baer and p.p.-Rings,” Journal of the Australian Mathematical Society, Vol. 18, No. 4, 1974, pp. 470-473. http://dx.doi.org/10.1017/S1446788700029190

[2] M. B. Rege and S. Chhawchharia, "Armendariz Rings," Proceedings of the Japan Academy, Series A, Mathematical Sciences, Vol. 73, No. 1, 1997, pp. 14-17. http://dx.doi.org/10.3792/pjaa.73.14

[3] D. D. Anderson and V. Camillo, “Armendariz Rings and
Gaussian Rings," Communications in Algebra, Vol. 26, No. 7, 1998, pp. 2265-2272. http://dx.doi.org/10.1080/00927879808826274

[4] N. K. Kim and Y. Lee, “Armendariz Rings and Reduced Rings,” Journal of Algebra, Vol. 223, No. 2, 2000, pp. 477-488. http://dx.doi.org/10.1006/jabr.1999.8017

[5] C. Huh, Y. Lee and A. Smoktunowicz, "Armendariz Rings and Semicommutative Rings," Communications in Algebra, Vol. 30, No. 2, 2002, pp. 751-761. http://dx.doi.org/10.1006/jabr.1999.8017

[6] T. K. Lee and T. L. Wong, "On Armendariz Rings," Houston Journal of Mathematics, Vol. 29, No. 3, 2003, pp. 583-593.

[7] Y. Suiyi, “On the Extension of Armendariz Rings,” MS Thesis, Lanzhou University, Lanzhou, 2008.

[8] R. Antoine, "Nilpotent Elements and Armendariz Rings," Journal of Algebra, Vol. 319, No. 8, 2008, pp. 3128-3140. http://dx.doi.org/10.1016/j.jalgebra.2008.01.019

[9] Z. K. Liu and R. Y. Zhao, “On Weak Armendariz Rings," Communications in Algebra, Vol. 34, No. 7, 2006, pp. 2607-2616. http://dx.doi.org/10.1080/0092787060065139 8

[10] H. F. Wu, “On Weak 3-Armendariz Rings," Journal of Hangzhou Normal University (Natural Science Edition), Vol. 11, No. 3, 2012, pp. 241-244.

[11] H. F. Wu, "Extensions of Reduced Rings," Journal of Hangzhou Normal University (Natural Science Edition), Vol. 10, No. 5, 2011, pp. 407-410.

[12] J. C. McConnell and J. C. Robson, "Noncommutative Noetherian Rings,” A Wiley-Interscience Publication, John Wiley and Sons, Ltd., Chichester, 1987. 